\title{
CAPACITAÇÃO DE EQUIPES PARA INQUÉRITO SOROLÓGICO CANINO PARA PESQUISA DE LEISHMANIOSE VISCERAL
}

\section{Capacitation of canine serological survey team to research visceral Leishmaniasis}

*André Luiz Baptista Galvão1, Carla Daniela Dan De Nardo², Halim Atique Neto², Aline do Nascimento Benitez ${ }^{3}$, Cássio Ricardo Ribeiro ${ }^{4}$, Katia Denise Saraiva Bresciani ${ }^{1}$, Vanessa Leyko Aoki $^{5}$, Izalco Nuremberg Penha dos Santos ${ }^{5}$

\begin{abstract}
${ }^{1}$ Departamento de Apoio, Produção e Saúde Animal do Curso de Pós-Graduação em Medicina Veterinária, Faculdade de Medicina Veterinária - Universidade Estadual Paulista (FMVA/UNESP), Araçatuba - SP, ${ }^{2}$ Centro Universitário de Rio Preto (UNIRP), ${ }^{3}$ Universidade Estadual de Campinas (UNICAMP), ${ }^{4}$ Conselho Federal de Medicina Veterinária, Comissão de Bem-estar Animal do CRMVDF, ${ }^{5}$ Secretaria de Saúde, Centro de Controle de Zoonoses, Prefeitura de São José do Rio Preto -
\end{abstract} SP.

*Autor para correspondência: andrelgalvao@hotmail.com

RESUMO: A Leishmaniose Visceral (LV) é uma enfermidade grave, e quando não tratada pode ocasionar letalidade de $95 \%$ nos humanos. Em cães, há uma prevalência significativa, com muito dos casos assintomáticos. Diante de tal importância, o diagnóstico precoce em humanos e cães se faz necessário para mapeamento e controle da enfermidade nos municípios. No estado de São Paulo, o primeiro caso desta enfermidade, foi notificado em Araçatuba, em 1998. Desde então, têm ocorrido o avanço da doença no estado principalmente no Noroeste Paulista. Perante tal preocupação, a Prefeitura do município de São José do Rio Preto realizou um inquérito sorológico canino para pesquisa de LV no primeiro semestre de 2017. Para composição das equipes, o inquérito obteve a participação dos alunos do curso de medicina veterinária do Centro Universitário de Rio Preto (UNIRP). Com a finalidade de realizar este inquérito sorológico assegurando o bemestar animal, a qualidade da amostra para fins em diagnóstico e a educação em saúde foi realizado o treinamento das equipes. Logo, com este trabalho objetiva-se descrever a metodologia de capacitação das equipes para tal inquérito. A capacitação foi realizada por meio de orientação coletiva para descrição e explanação teórica, e supervisão individualizada na ação prática utilizando manequins de cães. $\mathrm{O}$ aprendizado foi aplicado por vinte alunos, de abril a maio de 2017, em tutores e em seus cães, residentes na área urbana do município. Foram coletadas 1.581 amostras de sangue de cães. Não foram registradas inconformidades que oferecessem risco ao bem estar animal e ou humano durante a ação. O treinamento otimizou o tempo para coleta de amostras de sangue de cães em área urbana como estratégia de saúde pública.

Palavras-chave: contenção; manequim; sangue.

ABSTRACT: Visceral Leishmaniasis $(\mathrm{VL})$ is a serious disease and, when not treated can cause $95 \%$ death in humans. There is a high prevalence in dogs, with many asymptomatic cases. Early diagnosis in humans and dogs is necessary to map and control this disease in the municipalities. In São Paulo state, the first case was reported in Araçatuba in 1998. Since then, the disease spread especially in the northwest of the state. Due to this concern, São José do Rio Preto City Hall carried out a canine serological survey to research the VL in the first semester of 2017. The students from the Veterinary School of Centro Universitário de Rio Preto (UNIRP) 
composed the serological survey teams. The training of the survey teams was essential to assure the animal well-being, the quality of the samples used for diagnosis, and health information to the population. The present work aims to describe the methodology used for the training of the survey teams. The capacitation was done through theoretical explanation and description of the methods. The practice was carried out by supervised individual orientation using canine mannequins. Twenty students from the survey team taught tutors and their dogs, residents in the urban area of the municipality, from April to May 2017. A thousand five hundred and eighty one blood samples from the dogs were collected. There were no violations registered that could present any risk to the animals or humans well-being during the action. The training optimized the time to collect blood samples from dogs in urban area as public health strategy.

Keywords: constraint; blood; mannequin. 


\section{INTRODUÇÃO}

A leishmaniose visceral (LV), também denominada de leishmaniose visceral canina (LVC) trata-se de uma zoonose registrada em diversas regiões do mundo, que apresenta variações no ciclo epidemiológico, conforme a espécie do agente etiológico, a região geográfica em questão e as espécies de mamíferos susceptíveis que são expostas ao risco da infecção (Alves e Bevilacqua, 2004).

Esta parasitose é causada por um protozoário da ordem Kinetoplastida, família Trypanosomatidae, gênero Leishmania Ross 1903, espécie Leishmania (Leishmania) infantum chagasi (Lainson e Rangel, 2005), sinonímia Leishmania (Leishmania) infantum, que é transmitida aos seres humanos, animais silvestres (raposas, marsupiais, gambás, cachorros vinagre assim como roedores) e cães domésticos por meio da picada de flebotomíneos vetores (WHO, 2010). Dentre os reservatórios, o cão doméstico é considerado o principal reservatório para os humanos, sobretudo em áreas urbanizadas (Genaro, 2000; Alves e Bevilacqua, 2004; Dantas-Torres et al., 2012).

No estado de São Paulo, a LVC passou a constituir um grave problema de Saúde Pública, a partir de 1998, devido a uma epidemia diagnosticada na região de Araçatuba, região Oeste do Estado (Camargo-Neves et al., 2001, Brazil, 2013). A partir desta área, foram identificadas outras regiões comprometidas, constatando-se a dispersão da infecção devido à circulação indiscriminada de cães infectados e à manutenção de condições ambientais favoráveis à proliferação do vetor (Rangel et al., 2013). Diante de tal situação, a prefeitura do município de São José do Rio Preto -SP junto ao centro de controle de zoonoses com a secretaria de saúde do município realizou inquérito sorológico canino para pesquisa de LVC durante o período de 27 de março a 27 de abril de 2017. Para a formação das equipes, 0 inquérito obteve a participação dos alunos do curso de medicina veterinária do Centro Universitário de Rio Preto (UNIRP).

Com os objetivos centrados na importância da LVC em saúde pública, na relevância da educação em saúde e no conceito de bem-estar animal, foi então, realizado 0 treinamento das equipes, constituindo na descrição da metodologia empregada para tal capacitação o escopo do presente trabalho.

\section{MATERIAL E MÉTODOS}

\section{Laboratórios e equipes}

A capacitação das equipes foi realizada nas dependências do Hospital Veterinário "Dr. Halim Atique" - Centro Universitário de Rio Preto (UNIRP) São José do Rio Preto - SP. No total, foram capacitados cinco grupos de quatro alunos do terceiro ao sétimo período do curso de Medicina Veterinária do UNIRP. Adicionalmente, dois professores graduados em medicina veterinária, um com titulação de mestre e outro de doutor, foram os orientadores de todo treinamento, cuja parte prática durou quatro horas e foi realizada sete dias antes do início do inquérito sorológico. Tal capacitação das equipes foi previamente aprovada pela supervisão do Hospital Veterinário "Dr. Halim Atique" - UNIRP.

Metodologia aplicada para capacitação do procedimento de coleta de amostras

Para a capacitação, foram recordadas a utilização de medidas de precauções padrão na enfermagem (Oliveira, 2016), ressaltando a importância da lavagem das mãos, o uso de luvas, o correto manuseio de seringas $e$ agulhas assim como $o$ destino adequado de descarte de cada 
um dos itens. Ademais, foram recapituladas as estruturas anatômicas e foram demonstradas as formas de inspeção, abordagem, contenção física mínima e vias de acesso venoso do cão. Todas essas atividades foram simuladas com manequins, como recurso didático.

A orientação da inspeção dos animais foi realizada seguindo a metodologia semiológica (Feitosa, 2014), que consiste em: (a) estado de consciência: alerta (normal), diminuído (deprimido, apático), aumentado (excitado, bravo); (b) características respiratórias (eupneia); (c) coloração de mucosas: normocorada, perlácea, cianótica, etc.; (d) postura e locomoção: normal ou anormal; (e) condição física ou corporal: obeso, gordo, normal, magro, caquético; (f) a inspeção da cabeça na avaliação das narinas, olhos, pavilhões auriculares e musculatura mastigatória; (g) estado do pelame: pelos limpos, brilhantes ou eriçados, presença de ectoparasitas (carrapatos, piolhos, pulgas, etc.); (h) forma abdominal: normal, anormal (ascite, etc.). Estas instruções básicas foram importantes neste treinamento e visaram o bem-estar do cão.

Portanto, caso perante em tal inspeção prévia ao momento da coleta de amostra de sangue, o cão indicasse sinais específicos de doença neurológica e/ou cardiorrespiratória descompensada, desconforto e/ou riscos, a orientação era de que o procedimento de coleta de amostra de sangue não fosse realizado e, que o cão fosse conduzido para um atendimento médico veterinário de melhor suporte (Camacho e Mucha, 2014; Artero, 2019). Ademais, nesses casos, a orientação passada aos alunos, foi que a coleta de amostra de sangue para o inquérito, fosse agendada após a estabilização clínica do cão. Outra recomendação transmitida aos alunos foi que todas as etapas do procedimento de coleta de amostras de sangue fossem efetuadas em local apropriado e seguro no domicílio do cão, seguindo as recomendações de bem-estar e minimizando qualquer tipo de estresse ao animal (Broom e Fraser, 2007; Artero, 2019).

A simulação da socialização com o cão foi realizada com 0 auxílio de manequins; os alunos foram orientados na utilização da fala em tom amistoso, passando a mão sobre o dorso do cão, dando-lhe, posteriormente, as costas da mão para cheirar. A forma de contenção foi aplicada nos manequins mantendoos sobre uma mesa de superfície não escorregadia, após a colocação da mordaça ou de uma focinheira (Figura 1 A), o uso do colar elisabetano como auxílio na contenção também foi empregado. Posteriormente, foi realizada a demonstração da imobilização manual em posição quadrupedal e em decúbito lateral para melhor demonstração das vias de acesso para coleta de sangue (Figura 1B) (Feitosa, 2014).

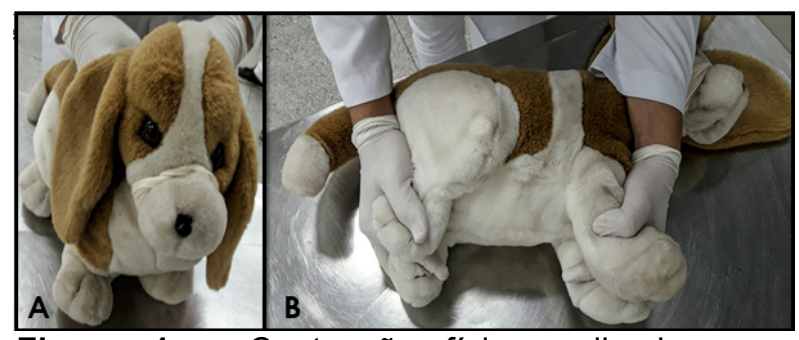

Figura 1 - Contenção física aplicada nos manequins, mostrando a colocação de mordaça (A) e contenção em decúbito lateral (B).

Para a demonstração e realização do procedimento de venopunção e coleta de sangue, foram utilizados três manequins de material de algodão e poliéster com a mesma disposição anatômica das veias que do cão em modelo vivo. Também foram utilizados sete modelos em manequins de isopor, tubo em látex e malha elástica de disposição anatômica similar do membro torácico e apresentação da veia cefálica. Para simulação do conteúdo correspondente ao sangue, 
em coloração vermelha, junto a estes manequins foram acoplados equipos macrogotas com frascos de $250 \mathrm{~mL}$ de solução de $\mathrm{NaCl} \quad 0,9 \%$ com tinta vermelha diluída. Para tanto, aos alunos foram fornecidos os manequins, tubos de látex de espessura fina e textura lisa; algodão com álcool etílico hidratado $70 \%$; seringas com volume de $3 \mathrm{~mL}$ e agulhas hipodérmicas $25 \times 7$. No procedimento de execução no manequim de cão, o mesmo foi contido, seguido então da colocação na circunferência do membro torácico ou pélvico de um tubo de látex em posição anatômica correspondente a veia cefálica ou safena, para simular a parada da passagem de sangue, para a posterior venopunção e coleta de sangue (Figura 2). Após este procedimento, foi então realizada a passagem do algodão com álcool etílico hidratado $70 \%$ no manequim, com o objetivo de desgerminar, seguido de identificação do vaso sanguíneo e posterior coleta de material por acesso intravenoso com agulha e seringa (Figura 3), simulando a condição real (Taylor, 2010). O volume de amostra obtido foi de aproximadamente $3 \mathrm{~mL}$.

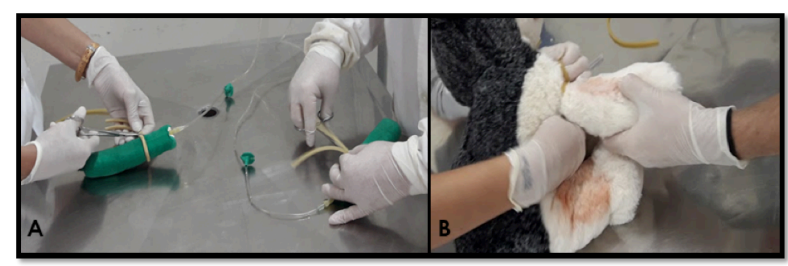

Figura 2 - (A) Colocação de um tubo de látex em posição anatômica correspondente a veia cefálica, na circunferência do manequim simulador do membro torácico, para simular a parada da passagem de sangue, para a posterior venopunção e coleta de amostra. (B) $O$ mesmo procedimento é mostrado no manequim.

\section{Educação em saúde}

O treinamento também foi direcionado nas medidas de prevenção de controle da LVC, por meio da orientação aos alunos de como proceder na explicação sobre a doença e distribuição de um panfleto explicativo para os tutores (Figura 4), contendo informações de controle e cuidados sobre a LVC.

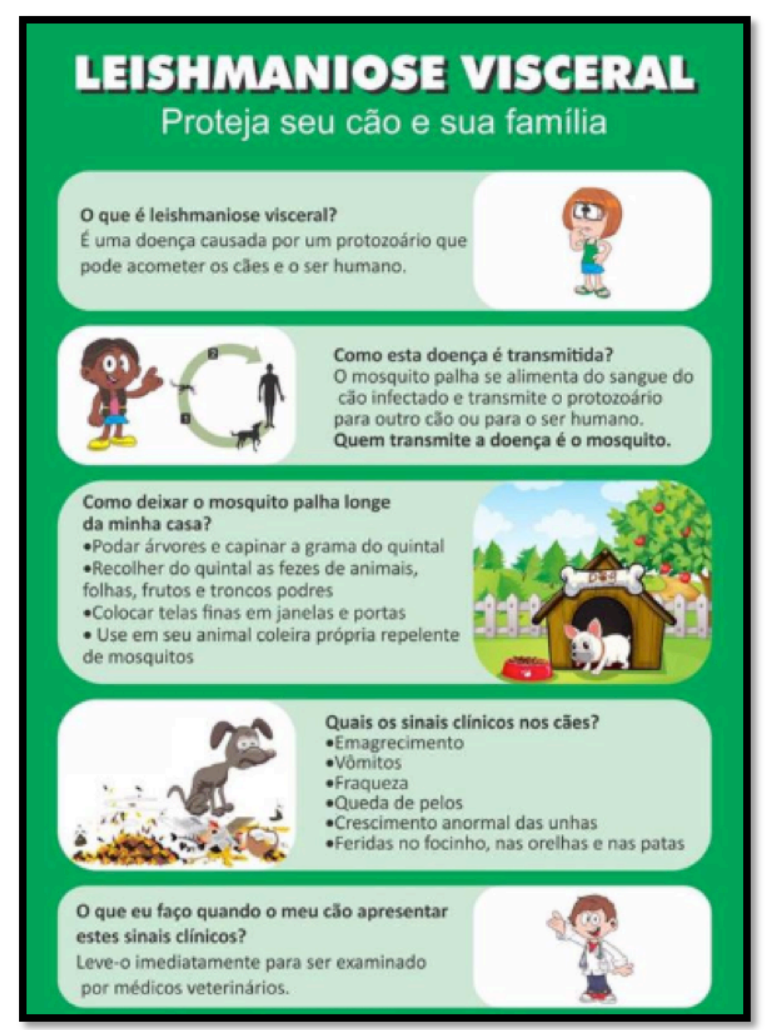

Figura 4 - Panfleto explicativo entregue ao tutor, contendo informações de controle e cuidados sobre a LVC.

\section{RESULTADOS E DISCUSSÃO}

A educação apresentada na forma da abordagem ao tutor e ao cão, bem como o treinamento das etapas do procedimento de venopunção e coleta de amostra de sangue são métodos necessários para a correta segurança de todos (Calderón-Maldonado e Garcia, 2015). Os animais domésticos não possuem a capacidade de se comunicar verbalmente informando desconforto e/ou dor (Broom e Fraser, 2007). Desse modo, o conhecimento da inspeção, abordagem e cuidados na contenção são de grande importância para o bem-estar e saúde do animal, tal como para a qualidade da amostra de sangue obtida (Broom e Fraser, 2007; Feitosa, 2014). Os objetivos da capacitação do presente trabalho, estavam centrados nas formas de 
inspeção, abordagem, contenção para execução de coleta de amostras de sangue realizadas com segurança, utilizando manequins como simuladores de cães, tais medidas permitiram preparo prévio para o inquérito, ademais, tais cuidados de instrução visaram na diminuição da ocorrência de fugas $e$ acidentes durante $o$ procedimento de coleta de amostra. Ademais, foi orientado aos alunos que durante a inspeção dos cães, sinais de comprometimento do sistema nervoso e cardiorrespiratório exigiam cuidados veterinários especiais (Artero, 2019) não sendo indicado nesses casos a coleta de sangue, entretanto, não houve registros de cães com alterações clínicas que impossibilitasse 0 procedimento de coleta de sangue.

No período de 27 de março a 27 de abril de 2017, com o auxílio dos alunos do curso de Medicina Veterinária do UNIRP, foram coletadas 1.581 amostras de sangue de cães para fins em diagnóstico da LVC para o inquérito sorológico do município de São José do Rio Preto-SP. Com a referida capacitação das equipes, os alunos demonstraram aptidão no procedimento de venopunção e coleta de amostras de sangue em cães.

No contexto didático, a oportunidade de tal treinamento para o aluno estimulou sua participação, formas de diálogo e seu o pensamento crítico. Metodologias de ensino como estas que foram realizadas na capacitação podem ser consideradas como excelentes formas de aprendizado (Madeira, 2010), pois simulam situações práticas, com uso de habilidade manual e permitem aos alunos um melhor aproveitamento, desenvolvimento e enriquecimento como futuro profissional.

Para o procedimento das coletas de amostras sangue, os alunos foram orientados que as mesmas fossem realizadas em ambiente apropriado ao animal, sendo considerado estado de higidez e saúde do cão como quesitos primordiais. Estas condições foram similares a estudos anteriores (Fraser, 2008) como medidas importantes para o bem-estar animal.

A adoção de tal capacitação foi realizada nas equipes do presente trabalho, com a instrução de medidas preventivas adequadas passadas a população do município, com o auxílio de um panfleto informativo. Considerando que no Brasil, onde a LV é um sério problema de saúde pública (Brazil, 2013), a e educação em saúde precisa estar implantada em todos os serviços públicos que desenvolvem as ações de controle da LVC, principalmente com a capacitação das equipes envolvidas em inquéritos (Brasil, 2014). Do mesmo modo, as ações de educação em saúde e de mobilização social representam um papel importante no campo da vigilância e controle da LVC, na medida em que se constitui de potencial transformador na construção de novas ferramentas para à prevenção e controle desta enfermidade (Rangel et al. 2013).

\section{CONCLUSÃO}

A metodologia empregada na referida capacitação mostrou-se adequada aos objetivos propostos. Os alunos do curso de medicina veterinária do UNIRP que participaram do treinamento demonstraram-se aptos para o procedimento de coleta de sangue em cães para o referido inquérito. Adicionalmente, tal metodologia aplicada foi de grande valor, pois contribuiu no aprendizado e formação dos alunos como profissionais, além do aprendizado com o envolvimento na ação de educação em saúde e de mobilização social no controle da LVC. 


\section{ACKNOWLEDGMENTS}

Aos profissionais do Centro de Controle de Zoonoses e da Secretaria de Saúde do Município de São José do Rio Preto - SP envolvidos nesta ação.

À supervisão do Hospital Veterinário "Dr. Halim Atique" - Centro Universitário de Rio Preto (UNIRP) pela concessão do espaço físico e disponibilidade do material de consumo para tal capacitação das equipes.

À coordenação do Curso de Medicina Veterinária do UNIRP pelo apoio nesta ação.

À Profa. M.e. Rosely Domingues e ao designer gráfico João Candido de Carvalho, ambos do Departamento de Comunicação Social do UNIRP pelo auxílio na elaboração e arte do panfleto explicativo.

Aos médicos veterinários Jeferson Bazotti e Alessandra Cetrone pela proatividade, bem como ao laboratório MSD - Saúde Animal pelo apoio e auxílio técnico neste treinamento.

Aos professores, aprimorandos e funcionários do Hospital Veterinário "Dr. Halim Atique" - UNIRP que de alguma forma participaram diretamente ou indiretamente nesta capacitação.

\section{REFERÊNCIAS}

ALVES, W. A.; BEVILACQUA, P. D. Reflexões sobre a qualidade do diagnóstico da leishmaniose visceral canina em inquéritos epidemiológicos: o caso da epidemia de Belo Horizonte, Minas Gerais, Brasil, 1993-1997. Caderno de Saúde Pública, v. 20, n.1, p. 259-265, Jan-Fev 2004.

ARTERO, C. T. Guia rápido de emergências em pequenos animais. São Paulo: Medvet, 2019. 120p.

BRAZIL, R. P. The dispersion of Lutzomyia longipalpis in urban areas.
Revista da Sociedade Brasileira de Medicina Tropical. Uberaba, v. 46, n. 3, p.263-264, May-June 2013.

BRASIL. Ministério da Saúde. Secretaria de Vigilância em Saúde. Departamento de Vigilância Epidemiologica. Manual de vigilância e controle da leishmaniose visceral. Ministério da Saúde. 2014; 1. ed., 5. reimpressão 01120.

BROOM D.M.; FRASER A. Domestic animal behavior and welfare. $4 \mathrm{a}$ ed. UK: CABI International; 2007. 448p.

CALDERÓN-MALDONADO

N.A.; GARCIA R.C.M. Bem-estar animal. In: JERICO M.M.; ANDRADE NETO J.P.; KOGIKA M.M. Tratado de Medicina Interna de Cães e Gatos. Seção C Comportamento e Direito Animal, São Paulo: Ed. Roca, v. 2, 2015, Cap. 255, p.2282-2287.

CAMACHO, A. A.; MUCHA C. J. Sistema Circulatório de Cães e Gatos. In: FEITOSA, F. L. F. Semiologia Veterinária. $3^{\circ}$ ed. São Paulo: Roca, 2014, Cap, 7, p. 241 -262.

CAMARGO-NEVES, V. L. F.; KATZ, G.; RODAS, L. A. C. et al. Utilização de ferramentas de análise espacial na vigilância epidemiológica de leishmaniose visceral americana Araçatuba, São Paulo, Brasil, 19981999. Caderno de Saúde Pública, Rio de Janeiro, v. 17, n. 5, p.1263-1267, Set.-Out. 2001.

DANTAS-TORRES, F.; SOLANOGALLEGO, L.; BANETH, G; R. et al. Canine leishmaniosis in the old and new worlds: unveiled similarities and differences. Trends in Parasitology. v. 28, n. 12, p. 531-538. Dec. 2012.

FEITOSA, F. L. F. Semiologia veterinária: a arte do diagnóstico. $3^{a}$ Edição, São Paulo, Ed. Roca, 2014. $627 p$.

GENARO, O. Leishmaniose Visceral Americana. In: NEVES, D. P., MELO, A. 
L.; GENARO, O.; LINARDI, P. M. Parasitologia Humana. Rio de Janeiro: Livraria Atheneu, 2000, Cap. 10, p. 5672.

LAINSON, R.; RANGEL, E. Lutzomyia longipalpis and the eco-epidemiology of American visceral leishmaniasis, with particular reference to Brazil - a review. Memorial Instituto Oswaldo Cruz, Rio de Janeiro, v. 100, n. 8, p. 811-827, Dec. 2005.

MADEIRA, M. C. Sou professor universitário; e agora? 2 ed. São Paulo: SARVIER, 2010. 219p.

OLIVEIRA, R. G. Blackbook Enfermagem. Belo Horizonte: Blackbook Editora, 2016.816p.

RANGEL, O.; HIRAMOTO, R. M.; HENRIQUES, L. F. et al. Classificação epidemiológica dos municípios segundo o Programa de Vigilância e Controle da Leishmaniose Visceral Americana no Estado de São Paulo, para 2013. Boletim Epidemiológico Paulista. v. 10, n. 111, p. 3-14. Mar. 2013. ISSN 1806-4272.

TAYLOR, S. M. Semiotécnica de pequenos animais. São Paulo:

Elsevier, 2011. 240p. 